\title{
Impact of configuration errors on the dynamic oscillation absorbers effectiveness of different masses on the seismic resistance of bridges
}

\author{
Ulugbek Shermuxamedov ${ }^{*}$ and Said Shaumarov ${ }^{\dagger}$ \\ Tashkent Institute of Railway Engineers, Adilkhodjaev st., 1, 100067, Uzbekistan
}

\begin{abstract}
Analyzes existing known solutions for dynamic damping oscillations of bridges in earthquakes. Substantiates the impact of errors settings the two-mass systems on the efficiency of dynamic dampers of different masses (small, large and commensurate). Based on the obtained of optimal parameters were built isolines depending reduce the displacement of the main mass of the setting on the stiffness and damping for different masses. The estimates obtained allow to simplify essentially the task of designing seismic devices for bridges.
\end{abstract}

\section{Introduction}

With significant advances in the calculation and design of various structures in seismic areas, the theory of seismic resistance of bridges lags behind the general theory of seismic resistance.

The existing recommendations on the design of bridges do not take into account the principles of multi-level design and new structures of antiseismic reinforcement of bridges based on the principles of seismic isolation and seismic suppression.

There is the construction of a great number of bridges with special earthquake protection solutions in the world's practice. One of the most promising earthquake protection techniques is the arrangement of seismic isolating bearing parts. In this case the achievement of dynamic oscillation damping due to usage of the framework weight is able to substantially increase the earthquake protection efficiency.

\section{Methods}

The paper is dedicated to the influence of adjustment accuracy on the application efficiency of dynamic dampers of various weights in the bridges. At present the dynamic oscillation dampers (DOD) have become a frequent practice in the seismic protection of structures. It is worthy of note that if flexibility and strength conditions of the elastic constraint are maintained, DOD proves to be the most efficient seismic protection system and allows decreasing the seismic load on the structure in several times and more. In particular, the

\footnotetext{
* Corresponding author: ulugbekjuve@ mail.ru

† Corresponding author: shoumarovss@gmail.com
} 
issues of DOD application to improve seismic resistance of the structures are developed by Korenev B.G., Polyakov V.S., Reznikov L.M., Uzdin A.M. and other specialists [4, 6].

The paper considers the influence of adjustment errors to the efficiency of dynamic dampers of various weights. Three types of dampers are mentioned as an instance:

- light weight damper - weights $10 \%$ of the protected structure weight $\left(v=\mathrm{M}_{\mathrm{damp}} / \mathrm{M}_{\mathrm{str}}=0.1\right)$;

- comparable weight damper, whose weight is equal to the structure weight $\left(v=\mathrm{M}_{\mathrm{damp}} / \mathrm{M}_{\mathrm{str}}=1\right)$

- large (supercritical) weight damper, the damper weight exceeds the critical weight, that is $v>v_{\mathrm{cr}} \approx 2$, and no traditional damping effect is observed.

The analysis of DOD technical solutions has shown that the material weakness of the light weight dampers, as mentioned in the papers of Savinov O.A. [1] and Nikitin A.A. [5], is the necessity of their high precision adjustment. Small deviations in the damper adjustment or its damping action invalidate its efficiency. In the early 1980s, Uzdin A.M. and Nikitin A.A. offered to use large weight dampers. Furthermore, in spite of their high efficiency the large weight dampers also have some specific difficulties for implementation. First, as known from [5], a critical weight exists. If the damper weight is larger than the structure weight more than twice, the damping effect disappears. The dynamic damper turns into a Lanchester damper. Herewith, the stiffness of elastic constraint becomes infinitely small but the damping action becomes considerable. Secondly, the damping effect often becomes insufficient and movements of the framework with regard to the supports become redundant causing the framework fall down from the supports. In particular, for the bridges this idea finds the application when the framework is used as the damping weight. Based on their investigations the theory of dimensionless equations for two-mass system was investigated (fig. 1, scheme "c") with optimum adjustment of stiffness of DOD ductile connections.

All the three cases have practical meaning. For example, to damp the building oscillations with usage of a flexible top level we obtain the damper weight about $10 \%$ of the protected structure weight. Though the weight of the damper in question is quite high it refers to the light weight DODs because its adjustment parameters can be estimated using the invariant points method [2] and optimized by the known formulae [3].

The second type of damper, at $v=1$, is already the comparable weight damper, for which the invariant points method is not applicable and the adjustment is done as per the formulae of Nikitin A.A. [7]. Implementation of parameters of such a damper, particularly of the friction parameter, may cause certain difficulties. Such dampers are typical, for example, in the earthquake protection of bridge piers when the steel span is used as the damping mass.

The third type of damper is the very large weight damper, in our example this is $v=5$. Such situation occurred in Sochi when during the construction of Adler-Sochi railway they used continuous concrete spans whose weight is entirely transferred on a single pier under a longitudinal load.

\section{Main part}

The efficiency of light weight, comparable weight and large weight DODs should be estimated by comparison with the reference systems. Herewith, the efficiency criterion is the decrease of maximum seismic load acting on a support by means of decreasing maximum movements of the pier under the seismic loads.

In the research of efficiency of the dynamic dampers for the bridge pier seismic oscillations the following DOD systems are considered as the reference systems:

1. Damper free system that is a pier without any DOD (fig.1, scheme "a"). 
2. The system where the damper is rigidly connected with a pier (scheme "b").

As the system in question we selected the scheme with dynamic damper as an additional weight which is resiliently connected with a pier (scheme "c").

a)

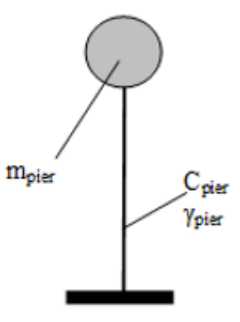

b)

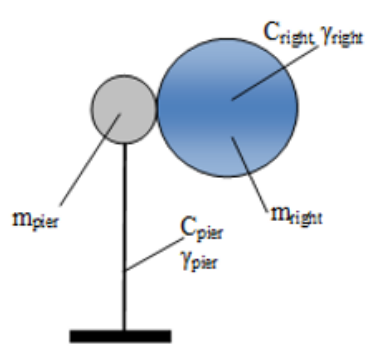

c)

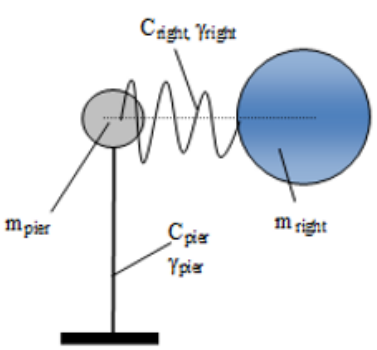

Fig. 1. Scheme of piers connected with the superstructure.

In the proposed scheme the following parameters are noted:

$\mathrm{C}_{\text {right }}-$ stiffness of damper (over-water length);

$\mathrm{C}_{\text {pier }}$ - stiffness of pier (landing bay);

$\mathrm{m}_{\text {right }}-$ mass of damper (over-water length);

$\mathrm{m}_{\text {pier }}-$ mass of pier (landing bay);

$\gamma_{\text {right }}-$ decay coefficient of damper (over-water length);

$\gamma_{\text {pier }}$ - decay coefficient of pier (landing bay).

The applied scheme "c" is calculated using the spectral technique and optimum stiffness and damping adjustment parameters are found.

To solve the assigned task, i.e. to analyze the incorrect adjustment influence on the efficiency of the damper performance the amplitude-frequency characteristics were analyzed. Amplitude-frequency characteristics were built using the formulae given in (1), (2), (3) for the optimum parameters of DOD.

Meanwhile the oscillation amplitude was calculated from the formula:

$$
\mathrm{U}_{\mathrm{s}}(\omega)=\sqrt{\mathbf{a}_{s}(\omega)^{2}+\mathbf{a}_{c}(\omega)^{2}}
$$

where $\mathbf{a}_{\mathbf{c}}$ and $\mathbf{a}_{\mathrm{s}}-$ are the unknown amplitude vectors at cosine and sine respectively,

$$
\begin{gathered}
\mathbf{a}_{s}=\left[\left(R-M \cdot \omega^{2}\right) \cdot B_{c}^{-1} \cdot\left(R-M \cdot \omega^{2}\right)+\mathbf{B}_{c}\right]^{-1} \cdot M \cdot V_{p} \cdot A \cdot g \\
\mathbf{a}_{c}=\left(R-M \cdot \omega^{2}\right)^{-1} \cdot \mathbf{B}_{c} \cdot \mathbf{a}_{s}
\end{gathered}
$$

$R, M$ and $B_{c}$ - are respectively the stiffness matrix, inertia matrix and damping matrix by Sorokin for the system in question.

\section{The optimal parameters of the system}

As the optimum we will consider such parameters at which the system displacements are the minimum in the given range of oscillation frequency.

The results of optimum parameters selection, i.e. for adjustment and damping for all the three cases are presented in fig. 2, 3 and 4, and optimum parameters for stiffness and damping were found using Builder $\mathrm{C}++$ and Mathcad software complex. 


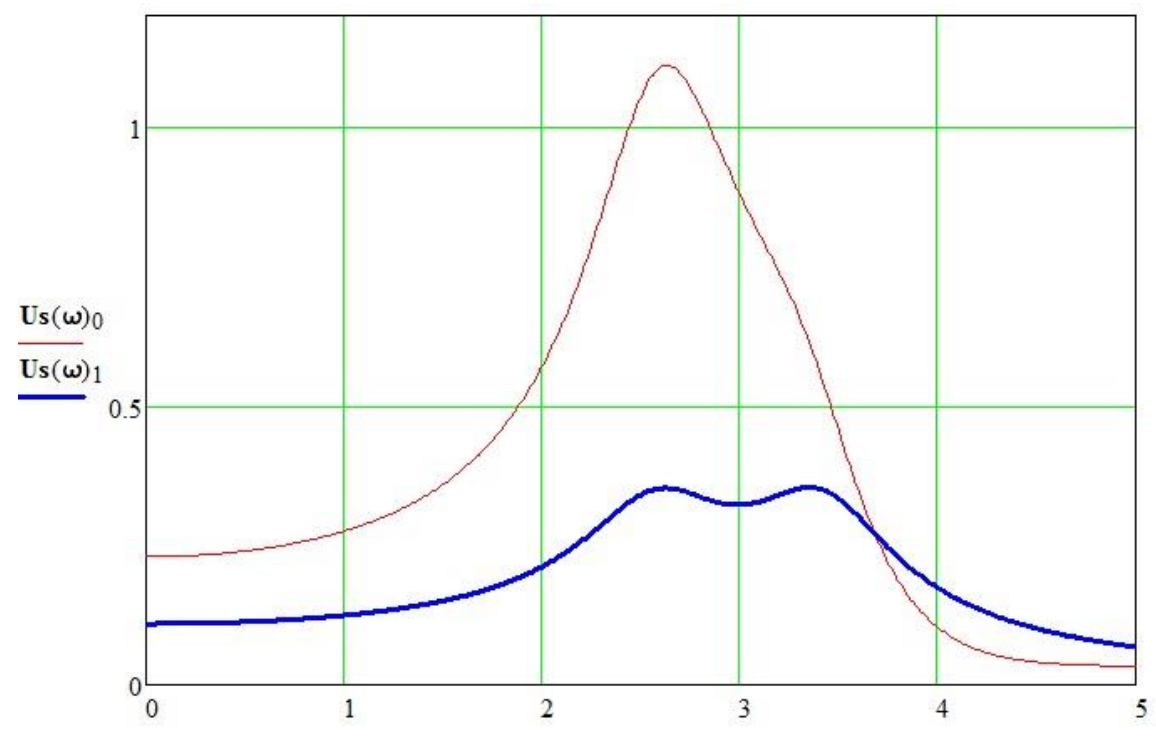

$\omega$

Fig. 2. Curve of amplitude-frequency characteristics versus disturbing frequency at the given parameters of the system $f_{1 \text { opt }}=0.86, \gamma_{1 \mathrm{opt}}=0.45$.



Fig. 3. Curve of amplitude-frequency characteristics versus disturbing frequency at the given parameters of the system $f_{3 \mathrm{opt}}=0.29, \gamma_{3 \mathrm{opt}}=3.1$. 


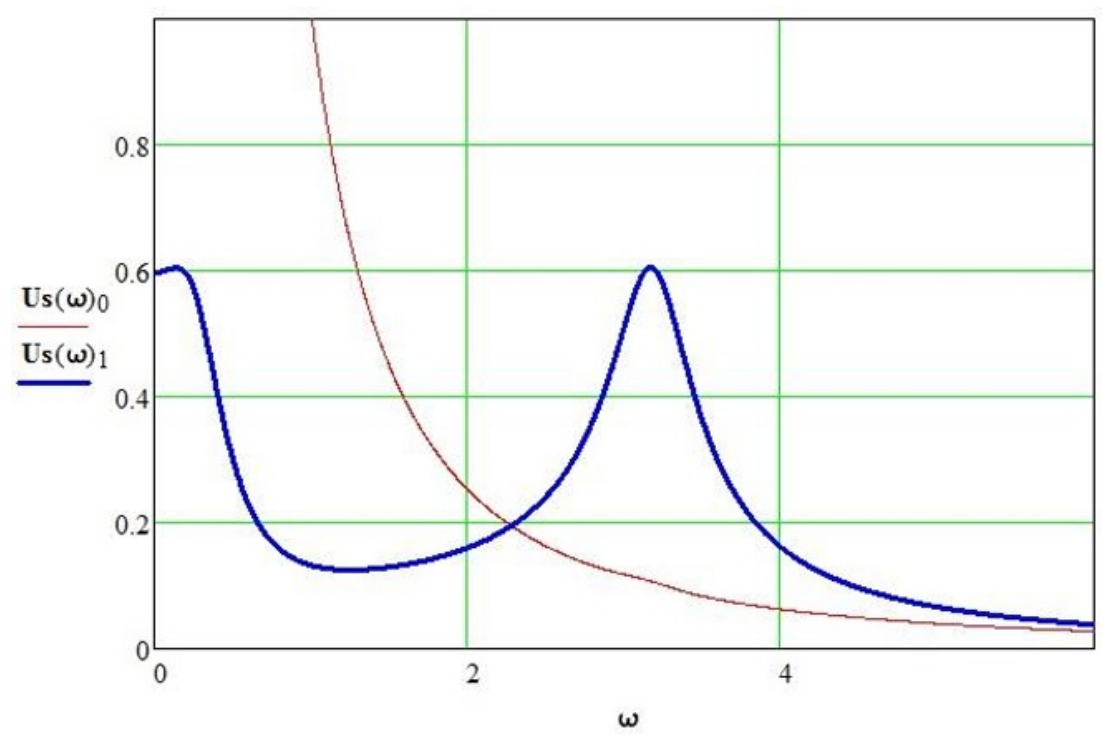

Fig. 4. Curve of amplitude-frequency characteristics versus disturbing frequency at the given parameters of the $\operatorname{system} f_{30 p t}=0.035, \gamma_{30 p t}=10.4$

From the above fig. 2, 3 and 4 it is clear that $\operatorname{Us}(\omega)_{0}$ and $\operatorname{Us}(\omega)_{1}$ are plotted on the vertical axis -the framework and pier displacement amplitudes, respectively, and $\omega-$ the disturbing frequency of fundamental harmonic of the pier oscillation is plotted on the horizontal axis.

The plots obtained were used to determine peak values of minimum displacements of the basic system. Then we studied the rate of change of the system displacement depending on the value of the damper adjustment inaccuracy.

\section{Isolines depending reducing displacement of the main mass of the setting for stiffness and damping}

To characterize efficiency of the damper adjustment the efficiency factor was introduced, which is algebraically expressed as follows:

$$
E\left(f, \gamma_{2}\right)=\frac{U_{\mathrm{opt}}-U\left(f, \gamma_{2}\right)}{U_{\mathrm{opt}}},
$$

where $U\left(f, \gamma_{2}\right)$ - extremum values of the system displacement function with adjustments $\mathrm{f}$ and $\gamma$ within the given frequency range; $U_{\text {opt }}$ - extremum values of the system displacement function with optimum adjustments.

From the formula (4) we obtained $E\left(f, \gamma_{2}\right)$ matrix, the curves of the main mass displacement decrease versus stiffness and damping adjustment in three cases $v=0.1, v=1$, $v=5$ (for light weight, comparable weight and large weight dampers respectively). Here the efficiency factor $U\left(f, \gamma_{2}\right)$, i.e. the efficiency of DOD application, can be compared with two major reference systems - the damper free system and the system where damper is rigidly connected with it, i.e. the system weight is increased by the damper weight value (see fig. 1). The figures 5, 6 and 7 demonstrate $E\left(f, \gamma_{2}\right)$ curves, where the system with rigid damper connection is applied as the basic one. 


\section{Conclusions}

For the large weight dampers, considerable deviations in the adjustment from the optimum are acceptable; moreover, the high efficiency zone substantially expands as the relative damper weight increases.

DOD system is more sensitive to the stiffness adjustment than to the damping adjustment. For example, for the case of $v=1$ the stiffness errors are acceptable within the range of $0.21<f<0.43$ and the damping errors - within the range of $1.4<\gamma_{2}<6.8$, at that the damping adjustment errors influence in a greater degree on the damping mass displacement and in a lesser degree on the main structure displacement.

The obtained estimations allow substantially simplifying the task of designing of the earthquake protection devices for bridges because they offer to a designer a wide possibility to choose the sizes and stiffness of the designed elements of the earthquake protection devices.

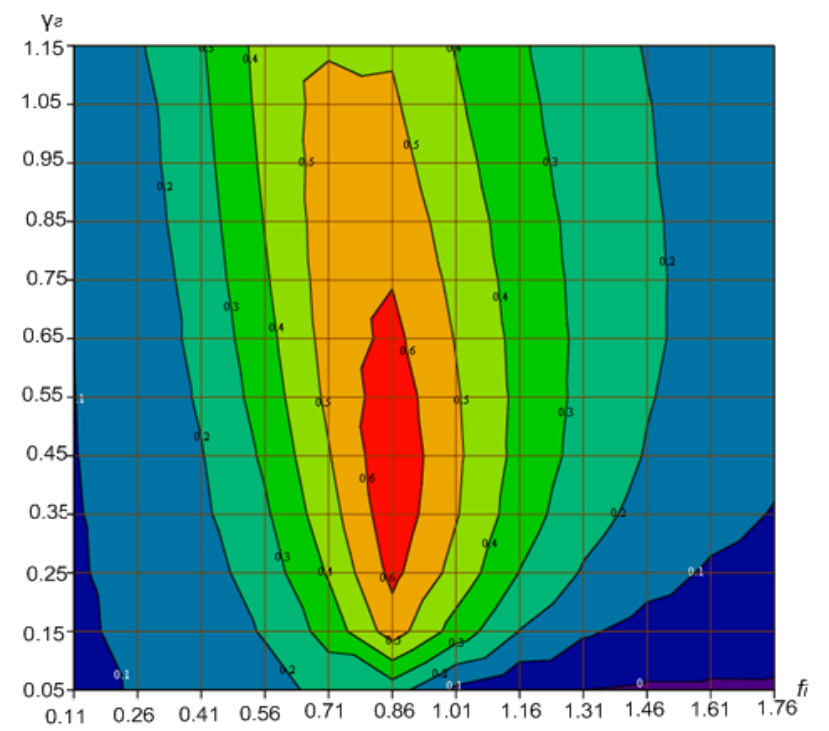

Fig. 5. Isoline of the main mass displacement decrease versus stiffness and damping adjustment at $v=0.1$. 


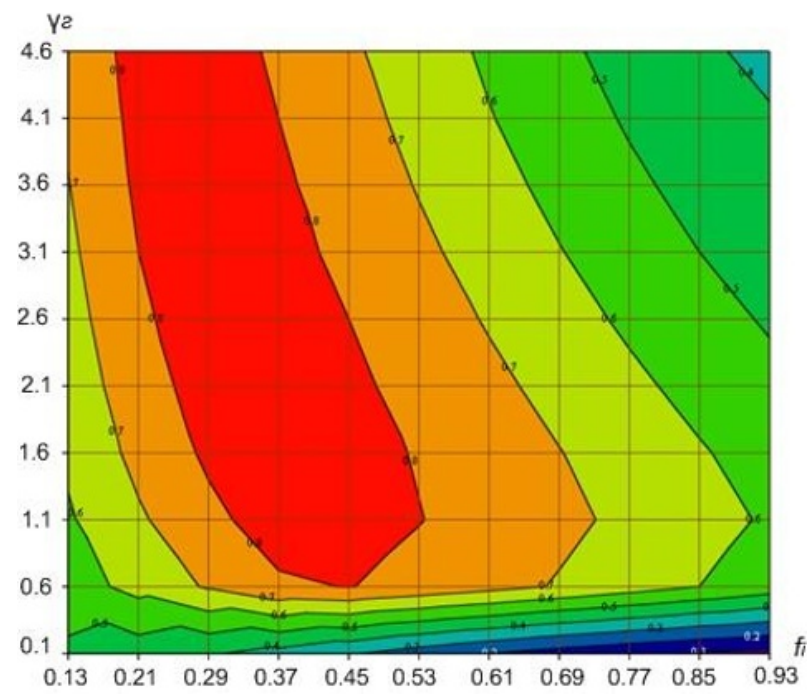

Fig. 6. Isoline of the main mass displacement decrease versus stiffness and damping adjustment at $v=1$.

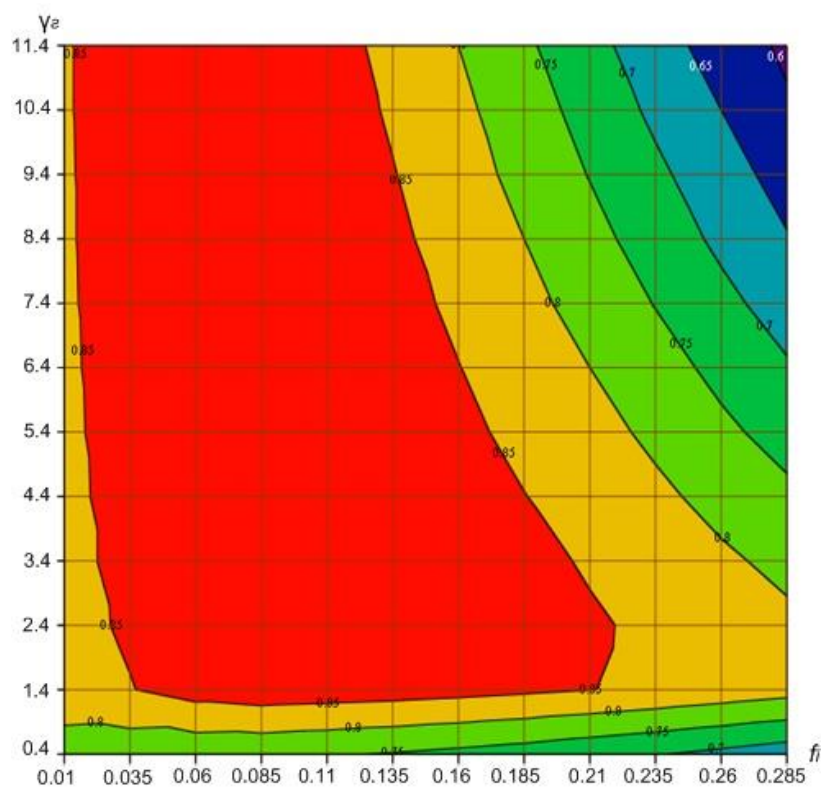

Fig. 7. Isoline of the main mass displacement decrease versus stiffness and damping adjustment at $v=5$.

\section{Reference}

1. Belash, T.A., Uzdin, A.M., Nikitin, A.A., Kuznetsova, I.O., Fedorova, M.Y., Gordeev, J.V., \& Dolgaya, A.A. Damping devices analysis for base isolated structure. PVPAmerican Society of Mechanical Engineers. Pressure Vessels and Piping Division Newsletter, 1998. 
2. Uzdin A.M., Kuznetsova I.O., Fedotova I.A. Soil mechanics and foundation engineering. - AA BALKEMA, 1997. - Vol. 2. - p. 1053-1056.

3. Savinov, O.A., Uzdin, A.M., Sandovich, T.A., Dolgaya, A.A. For earthquake proof foundation foundation designs. Soil Mechanics and Foundation Engineering, 32 (4), p.124-129.

4. Uzdin A.M., Nikitin A.A., Kuznetsova I.O. Application of Seismic Isolation for Transport and Hydrotechnical Structures in Russia // ASME-PUBLICATIONS-PVP. 1998. - T. 379. - p. 193-196.

5. Savinov O.A. et al. ESTIMATION OF FEATURES OF SEISMIC EFFECT AT THE SELECTION OF PARAMETERS OF SEISMOINSULATING CONSTRUCTIONS OF FOUNDATIONS // Grounds, foundations and soil mechanics. - 1995. - no. 4. - p. 9-13.

6. Kurbatskiy E.N., Minh M.D. FOUNDATION CALCULATION FOR BUILDINGS AND STRUCTURES WITH FOREIGNING AND CONSTRUCTIONS OF THE FOURIER TRANSFORMS FOR FI-NITE FUNCTIONS // Proceedings of Moscow State University. - 2014. - №. 1.

7. Savinov O.A. On the application of a dynamic oscillation damper // Proceedings of the research sector of the Leningrad branch of the trust of deep works. - L .: Stroyizdat Narkomstroya, 1940. - Vol. 1. - s. 30-35

8. Timoschenko S.P. Fluctuations in engineering. - M .: Science, 1976. - p. 209-216. [In Russian: Timoschenko, S.P. Oscillations in engineering. - M .: Science, 1976].

9. Korenev B.G., Reznikov L.M. Dynamic vibration dampers. - M .: Science, 1988. - 303 p.

10. Nikitin A.A., Uzdin A.M. The use of dynamic oscillation dampers for seismic protection of bridges // Express information VNIIIS. Ser.14. Earthquake resistant construction. - 1986. - Issue 9. - p.20-24

11. Simkin A.Yu., Nikitin A.A., Shulman S.A., Akhmedov T.Sh., Tkachenko S.S. Silnitsky Yu.M., Shestoperov G.S. The supporting part of the structure. A.S. USSR, MKI E01D 19/04, N 1162886, 1985.

12. Eliseev O.N, Uzdin A.M. Earthquake resistant construction. Textbook. SPb .: Ed. PVISU, 1997. - 371s. 